\title{
A CHARACTERIZATION OF COMMUTATIVE GROUP ALGEBRAS AND MEASURE ALGEBRAS
}

\author{
BY MARC A. RIEFFEL \\ Communicated by Walter Rudin, June 21, 1963
}

1. Introduction. The group algebra, $L(G)$, of a locally compact Abelian group, $G$, is the Banach algebra of all complex-valued functions on $G$ integrable with respect to Haar measure on $G$, with convolution as multiplication. The measure algebra, $M(G)$, of $G$ is the Banach algebra of all finite complex regular Borel measures on $G$, with convolution as multiplication.

In this note we give a characterization of those commutative Banach algebras which are the group algebras of locally compact Abelian groups, and a similar characterization of the measure algebras. Sketches of proofs will be given. Complete details will be published elsewhere.

The results reported in this note are contained in a dissertation submitted in partial fulfillment of the requirements for the Ph.D. at Columbia University. We are happy to have this opportunity to express our gratitude to Professor Richard V. Kadison for his encouragement of this work and for many helpful comments and suggestions.

2. Preliminaries. Define an abstract complex $L$-space, $B$, to be a partially ordered complex Banach space such that the real subspace, $R$, of $B$ generated by the positive elements of $B$ is a real Banach lattice under the order of $B$ restricted to $R$, and such that the following hold:

(I) If $x, y \in R$ and $x, y \geqq 0$, then $\|x+y\|=\|x\|+\|y\|$.

(II) If $x, y \in R$ and $x \wedge y=0$, then $\|x+y\|=\|x-y\|$.

(III) If $x \in B$, then there exist unique $\operatorname{Re}(x), \operatorname{Im}(x)$ in $R$ such that $x=\operatorname{Re}(x)+i \operatorname{Im}(x)$.

(IV) If for $x$ in $B$ we define $|x|=V\left\{\operatorname{Re}\left(e^{i \theta} x\right): \theta \in[0,2 \pi]\right\}$ then $\|x\|=\||x|\|$.

Then in analogy with Kakutani's theorem [5] on the concrete representation of abstract real $L$-spaces, $B$ is isometrically isomorphic to complex $L^{1}(X, m)$ for some measure space $(X, m)$.

Let $B$ be any Banach space, and let $f$ be any nonzero linear functional on $B$. Let $P(f)=\{x: f(x)=\|f\|\|x\|\}$. Then $P(f)$ is a cone in $B$ and so defines a partial order on $B$ in the usual way.

Now let $A$ be any complex commutative Banach algebra, with 
multiplication denoted by $*$, and let $f$ be a multiplicative linear functional (m.l.f.) on $A$. We say that $f$ is an $L^{\prime}$-inducing m.l.f. if under the order defined by $P(f) A$ is an abstract complex $L$-space, and if

(V) $\|f\|=1$.

(VI) If $x, y \in A$, then $|x * y| \leqq|x| *|y|$ where || is with respect to the order defined by $P(f)$.

3. Main result. Our characterization of group algebras is

THEOREM 1. Let $A$ be a commutative semi-simple Banach algebra such that

(1) every m.l.f. of $A$ is $L^{\prime}$-inducing,

(2) $A$ is Tauberian.

Then there exists a locally compact Abelian group, $G$, such that $A$ is isometrically isomorphic to $L(G)$.

Condition (2) means that the elements of $A$ whose Gel'fand transforms have compact support are dense in $A$. Part of the proof of Theorem 1 can be given the following more general setting

Theorem 2. Let $A$ be a complex commutative Banach algebra, and let $D$ be the collection of all $L^{\prime}$-inducing m.l.f. of $A$. Suppose that (1) $D$ is a separating family of linear functionals for $A$; then there exists a compact Abelian group, $B$, such that $A$ can be embedded in $M(B)$. If in addition (2) $D$ is locally compact in the w* topology, then there exists a locally compact Abelian group, $G$, whose Bohr compactification is $B$ and whose dual group is homeomorphic to $D$, such that $A$ can be embedded in $M(G)$.

Sketch of proof of Theorem 2. For a fixed $f$ in $D$ the complex analogue of Kakutani's theorem is used to represent $A$ as $L^{1}(X, m)$. The elements of $D$ are then elements of $L^{\infty}(X, m)$, and it is shown that as such $D$ is closed under pointwise multiplication and pointwise inverses, and so forms an Abelian group. Then the norm-closed linear span of $D$ in $L^{\infty}(X, m)$ is a sub- $C^{*}$-algebra of $L^{\infty}(X, m)$ and so is representable as $C(B)$ for some compact space $B$. The dual space of $C(B)$ is $M(B)$, and there is a natural map of $A$ into $M(B)$. The Kaplansky density theorem [2, p. 46] (we are indebted to E. Effros for bringing this theorem to our attention) ensures that this map is isometric. In analogy with an idea of Birtel [1] the multiplication in $A$ induces a Banach algebra multiplication in all of $M(B)$. If we identify points of $B$ with unit point measures, the multiplication in $M(B)$ induces a multiplication in $B$ for which $B$ is a compact semigroup with cancellation law. By a theorem given in $[4$, p. 814] and 
attributed there to Iwasawa, $B$ is then a group. The measure algebra of $B$ is $M(B)$ with the multiplication defined above. The dual group of $B$ is $D$ with the discrete topology. If condition (2) is satisfied, we let $G$ be the dual of $D$ with the $w^{*}$ topology, and use Eberlein's characterization of Fourier-Stieltjes transforms [3] to move $A$ from $M(B)$ to $M(G)$.

Sketch OF PROOF OF THEOREM 1. Theorem 2 is applied to obtain $G$, and then condition (2) is easily seen to imply that $A$ is a subalgebra of $L(G)$. A lengthy argument then shows that $A$ is all of $L(G)$.

We have an example which shows that condition (2) of Theorem 1 is necessary.

The characterization of measure algebras is accomplished by giving an abstract form of Eberlein's characterization of Fourier-Stieltjes transforms [3], and then applying Theorem 2.

\section{REFERENCES}

1. F. T. Birtel, On a commutative extension of a Banach algebra, Proc. Amer. Math. Soc. 13 (1962), 815-822.

2. J. Dixmier, Les algèbres d'opérateurs dans l'espace hilbertien, Gauthiers-Villars, Paris, 1957.

3. W. F. Eberlein, Characterizations of Fourier-Stieltjes transforms, Duke Math. J. 22 (1955), 465-468.

4. B. R. Gelbaum, G. K. Kalish and J. M. H. Olmsted, On the embedding of topological semigroups and integral domains, Proc. Amer. Math. Soc. 2 (1951), 807-821.

5. S. Kakutani, Concrete representations of abstract $(L)$-spaces and the mean ergodic theorem, Ann. of Math. (2) 42 (1941), 523-537.

Columbia University 